\title{
Recurrent huge leiomyoma of the urethra in a female patient: A case report
}

\author{
YUE-HONG SHEN and KAI YANG \\ Department of Urology, The First Affiliated Hospital, College of Medicine, Zhejiang University, \\ Hangzhou, Zhejiang 310003, P.R. China
}

Received August 29, 2013; Accepted February 2, 2014

DOI 10.3892/ol.2014.1991

\begin{abstract}
Urethral leiomyoma is an extremely rare condition that arises from the smooth muscle of the urethra. To the best of our knowledge, there is only a single reported recurrence treated by a repeat excision in the literature to date. The present study reports an exceptionally rare case of a recurrent huge leiomyoma of the female urethra. The 47-year-old female was diagnosed with a huge mass located between the urethra and vagina during a gynecological examination. The patient had no symptoms and was successfully treated with transabdominal excision. Pathological examinations revealed a leiomyoma of the urethra. The patient was followed up for one year without any sign of recurrence.
\end{abstract}

\section{Introduction}

Urethral leiomyomas are rare mesenchymal benign tumors of smooth muscle origin that occur almost exclusively in females (1). To the best of our knowledge, only $\sim 100$ cases have been reported in the literature (1-8). The etiology and pathogenesis of these tumors are unclear, although, some are hypothesized to have a hormonal influence $(5,6)$. Surgical excision is the first choice of treatment for these tumors and the prognosis has been good with the exception of one reported case of local recurrence (9). In the present study, a rare case of huge urethral leiomyoma recurrence in a Chinese female is reported. Patient provided written informed consent.

\section{Case report}

A 47-year-old Chinese female was admitted to the Department of Urology (The First Affiliated Hospital, College of Medicine, Zhejiang University, Hangzhou, Zheijiang, China), due to a

Correspondence to: Dr Kai Yang, Department of Urology, The First Affiliated Hospital, College of Medicine, Zhejiang University, 79 Qingchun Road, Hangzhou, Zhejiang 310003, P.R. China

E-mail: kaiyang@zju.edu.cn

Key words: leiomyoma, urethra solid mass detected during a gynecological examination. The patient was asymptomatic and had undergone two surgeries for urethral leiomyoma, which occurred six and nine years ago, respectively. Computed tomography demonstrated that the bladder and uterine were compressed by a $7.5 \times 7.0-\mathrm{cm}$ mass with well-defined outlines (Fig. 1). Magnetic resonance imaging was later performed to improve the definition of the structure and the association of the lesion with the urethra and vagina. The mass was isointense to muscle on T1-weighted images and slightly hyperintense on T2-weighted images, indicating a solid mass (Fig. 2).

The urethral tumor was completely excised and removed as close to the bladder neck as possible by transabdominal surgery. The detachment in the paraurethral region was meticulous to reduce injury to the urethra. The subsequent pathological diagnosis was of a leiomyoma. Immunohistological analysis demonstrated that the tumor cells were positive for desmin, cluster of differentiation 10 (CD10), smooth muscle actin and caldesmon, and negative for CD117 (Fig. 3). The patient was carefully followed up without any other treatment, and no sign of recurrence was observed in the first year post-surgery.

\section{Discussion}

Leiomyomas are benign tumors of smooth muscle origin that occur throughout the genitourinary system, most commonly in the renal capsule (7). Urethral leiomyomas are rare benign tumors affecting females significantly more than males (1-8). In 1894, Buttner (10) described the first urethral leiomyoma. Thus far, only $\sim 100$ cases have been reported in the literature (1-8). The posterior wall of the urethra is the site of predilection, although any wall may be affected (11). Additionally, the distal urethra can be affected, but the proximal segment is the most common site (12).

Urethral leiomyomas are usually asymptomatic when they are small. As they grow in size, patients may complain of urinary tract infection, dyspareunia, urinary retention or irritative lower urinary tract symptoms (13). Physical examination may reveal a mass in the anterior vaginal wall or one that protrudes from the urethral meatus (12). Ultrasonography and magnetic resonance imaging have been shown to provide useful pre-operative information regarding the morphology and structure of the mass $(14,15)$. However, a pathological 
A

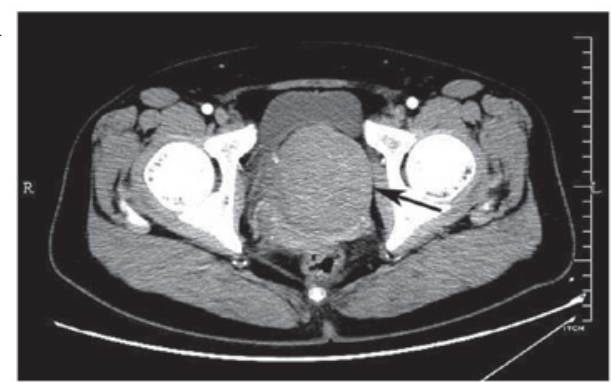

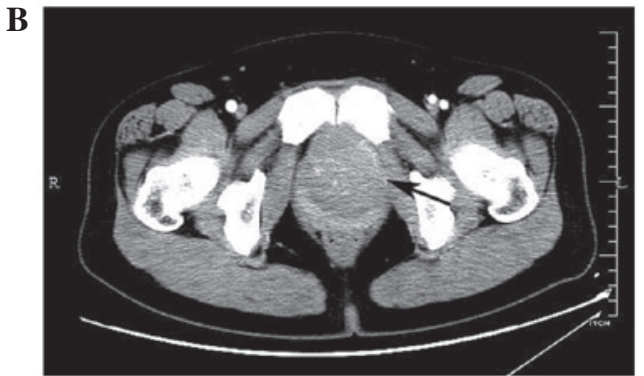

Figure 1. Contrast-enhanced computed tomography showing the presence of the tumor (A) compressing the bladder and uterine and (B) behind the pubic symphysis. Arrows indicate the tumor.
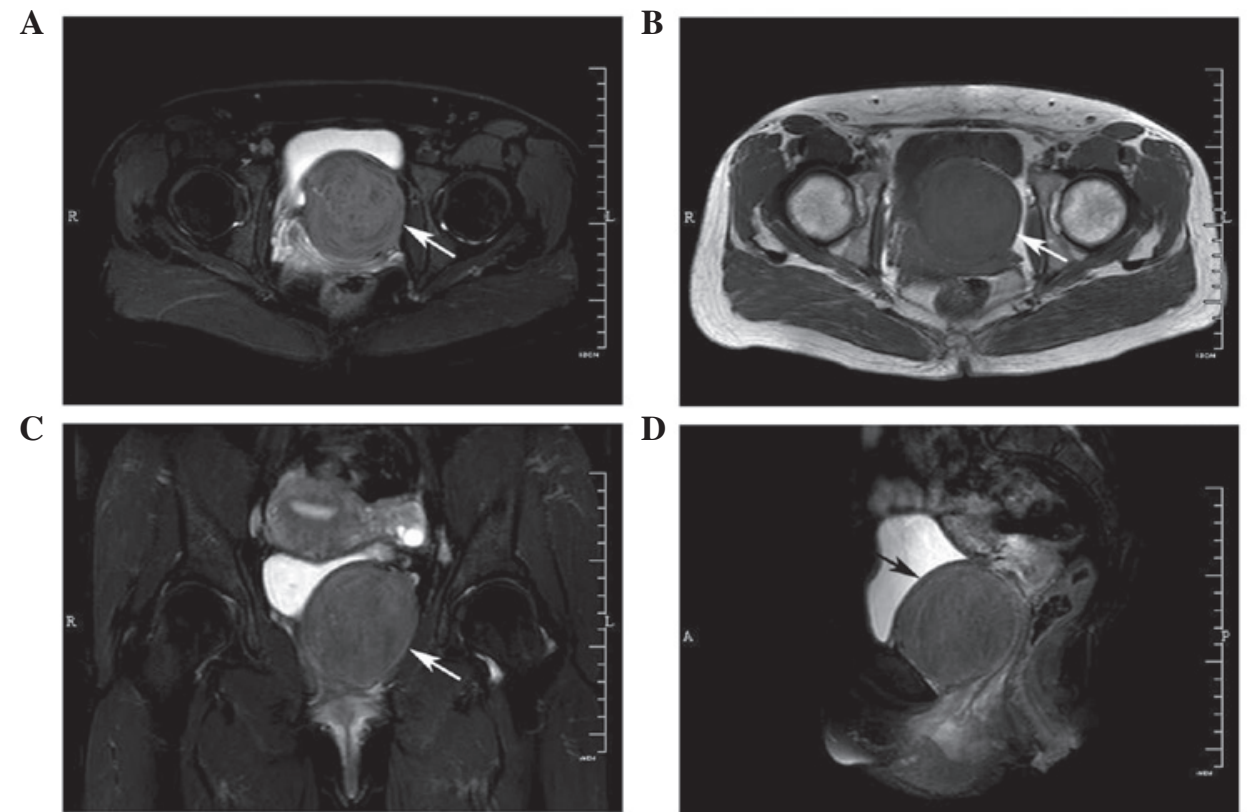

Figure 2. MRI showing the presence of the tumor on (A) T2-weighted and (B) T1-weighted images in the transverse plane. T2-weighted MRI showing (C) coronal and (D) sagittal planes. Arrows indicate the tumor. MRI, magnetic resonance imaging.
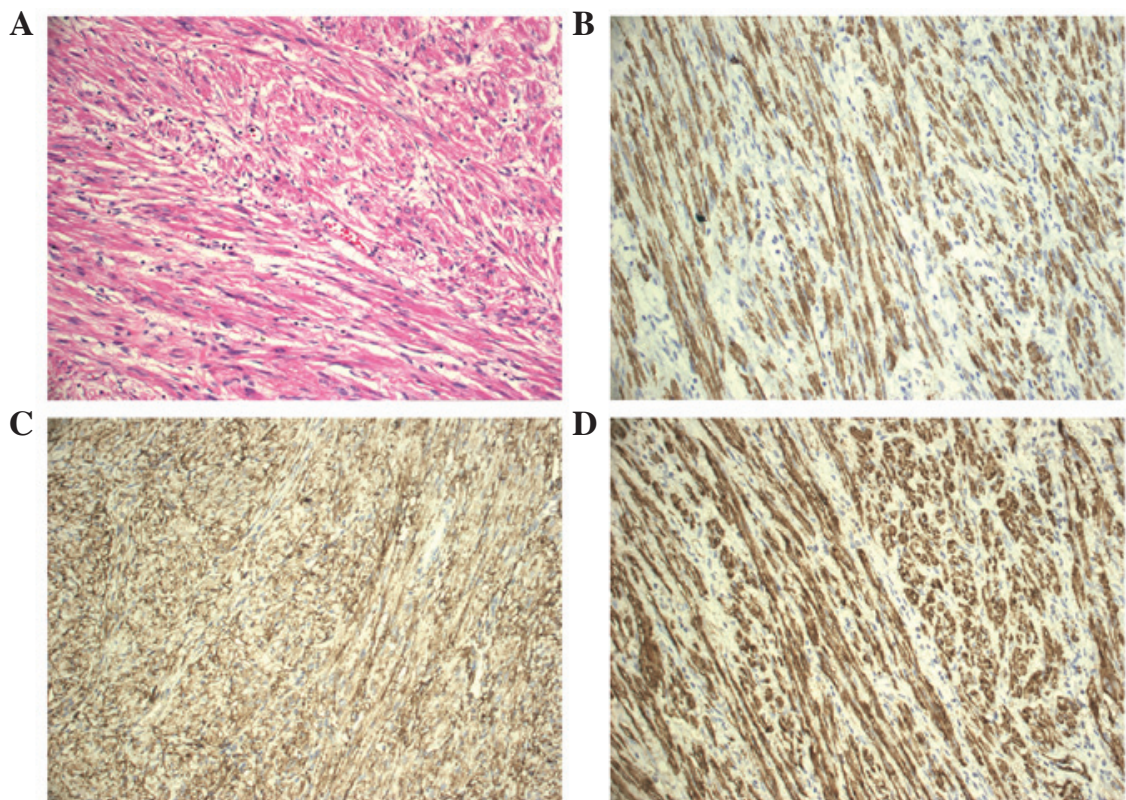

Figure 3. (A) Pathological results revealing the composition of the tumor, with spindle cells, a lack of nuclear atypia and mitotic figures (HE; magnification, $\mathrm{x} 200$ ). Immunohistochemical analysis showing positive cytoplasmic staining for (B) desmin, (C) CD10 and (D) caldesmon in the tumor cells (magnification, x200). HE, hematoxylin and eosin; CD10, cluster of differentiation 10. 
examination is indispensable to exclude the possibility of a malignancy.

In the present study, the huge pelvic tumor should be differentiated from the urethral carcinoma or masses that have originated from other tissues, although the patient had a past history of urethral leiomyoma present six and nine years ago, respectively. In all previously published cases, the urethral leiomyomas have been treated surgically, with surgical excision as the first choice of treatment. The present patient was treated by transabdominal excision and the tumor was found to originate from the proximal segment of the urethra, which is close to the bladder neck.

All previously reported vesical and urethral leiomyomas have followed a benign biological course (7), with only a single reported recurrence treated by a repeat excision (9). The present study is the second reported case of urethral leiomyoma recurrence, and the patient has undergone three surgical procedures to date.

\section{Acknowledgements}

This study was supported by grants from the National Natural Science Foundation of China (grant no. 81101718) and the National Key Clinical Specialty Construction Project of China.

\section{References}

1. Shield DE and Weiss RM: Leiomyoma of the female urethra. J Urol 109: 430-431, 1973.
2. Cheng C, Mac-Moune Lai F and Chan PS: Leiomyoma of the female urethra: a case report and review. J Urol 148: 1526-1527, 1992.

3. Leidinger RJ and Das S: Leiomyoma of the female urethra. A report of two cases. J Reprod Med 40: 229-231, 1995.

4. Leung YL, Lee F and Tam PC: Leiomyoma of female urethra causing acute urinary retention and acute renal failure. J Urol 158: 1911-1912 1997.

5. Alvarado-Cabrero I, Candanedo-González F and Sosa-Romero A: Leiomyoma of the urethra in a Mexican woman: a rare neoplasm associated with the expression of estrogen receptors by immunohistochemistry. Arch Med Res 32: 88-90, 2001.

6. Kato T, Kobayashi T, Ikeda R, Nakamura T, Akakura K, Hikage T and Inoue T: Urethral leiomyoma expressing estrogen receptors. Int J Urol 11: 573-575, 2004.

7. Goldman HB, McAchran SE and MacLennan GT: Leiomyoma of the urethra and bladder. J Urol 177: 1890, 2007.

8. Garrido Abad P, Fernández Arjona M, Herranz Fernández LM, Muñoz-Delgado Salmerón J and Capote LF: Leiomyoma of the male urethra: case report and review of the literature. Arch Esp Urol 63: 71-74, 2010.

9. Merrell RW and Brown HE: Recurrent urethral leiomyoma presenting as stress incontinence. Urology 17: 588-589, 1981.

10. Buttner: A case of myoma of the female urethra. Z Geburshe Gynak 28: 135, 1894 (In German).

11. Wani NA, Bhan BL, Guru AA and Garyali RK: Leiomyoma of the female urethra: a case report. J Urol 116: 120-121, 1976.

12. Lee MC, Lee SD, Kuo HT and Huang TW: Obstructive leiomyoma of the female urethra: report of a case. J Urol 153: 420-421, 1995.

13. Pahwa M, Saifee Y, Pahwa AR and Gupta M: Leiomyoma of the female urethra-a rare tumor: case report and review of the literature. Case Rep Urol 2012: 280816, 2012.

14. Pavlica P, Bartolone A, Gaudiano C and Barozzi L: Female paraurethral leiomyoma: ultrasonographic and magnetic resonance imaging findings. Acta Radiol 45: 796-798, 2004.

15. Ikeda R, Suga K and Suzuki K: MRI appearance of a leiomyoma of the female urethra. Clin Radiol 56: 76-79, 2001. 